\title{
Haemophilus influenzae pneumonia in Melanesian adults: report of 15 cases
}

\author{
DAVID J BARNES, SIRUS NARAQI, JOSEPH D IGO
}

From the Division of Medicine and Department of Pathology, Medical Faculty, University of Papua New Guinea

ABSTRACT In a prospective study of 170 adult patients with acute pneumonia, Haemophilus influenzae was found to be the aetiological agent in 15 cases $(8 \cdot 8 \%)$. The diagnosis in all cases was based on positive cultures of blood or percutaneous lung aspirate, or both. Chronic lung disease was significantly more common in patients with $H$ influenzae pneumonia than in patients with pneumonia due to other organisms but age, sex, and smoking history did not differ significantly. Lobar consolidation was the most common radiological pattern, being present in 10 of the 15 cases. Type b was the commonest serotype isolated, but three cases were due to non-typable (non-capsulate) strains. All patients survived, responding well to treatment with penicillin, ampicillin, or chloramphenicol. Haemophilus influenzae should be considered as a possible cause of pneumonia in adults, particularly those with underlying chronic lung disease.

\section{Introduction}

Haemophilus influenzae, a Gram negative bacillus or coccobacillus, is a frequent isolate from the upper respiratory tract of healthy individuals. ${ }^{1}$ It can be recovered from the nasopharynx of up to $80 \%$ of the normal population, and in smokers and patients with chronic bronchitis the trachea and major bronchi are frequently colonised. ${ }^{12}$ The organism exists with or without a polysaccharide capsule, and six antigenically distinguishable capsular types (designated $a-f)$ have been identified. The non-capsulate (nontypable) strains commonly cause luminal disease (bronchitis, sinusitis, otitis media) while most systemic or invasive disease is caused by capsulated strains, particularly type $b$. While the role of type $b$ in the aetiology of childhood pneumonia is well recognised, $H$ influenzae is generally thought to be an uncommon cause of acute pneumonia in adults. ${ }^{3-11}$

In a prospective study of adult pneumonia in Papua New Guinea we sought to determine the frequency of $H$ influenzae pneumonia and discover whether these patients had any distinctive clinical or radiological features.

Address for reprint requests: Dr David J Barnes, 26 Harris Street, Cronulla 2230, New South Wales, Australia.

Accepted 15 June 1987

\section{Methods}

All adults with pneumonia admitted to the University Hospital in Port Moresby, Papua New Guinea, during March-December 1986 were studied. The diagnosis of pneumonia was based on short duration of symptoms, consistent clinical signs, and the presence of an infiltrate on the chest radiograph. Particular attention was given to clinical features, radiological patterns, aetiology (bacterial and viral), and outcome. Bacteriological diagnosis was based on culture of blood and percutaneous lung aspirate. Sputum, when available, was collected for culture but no bacteriological diagnoses were made on the basis of these results alone. Serotyping of isolates of $H$ influenzae from blood and lung aspirate cultures was carried out with agglutinating antisera. Patients with pneumonia due to $H$ influenzae were compared with the total group of adults admitted with acute pneumonia.

Statistical analysis was performed with Yates' $\chi^{2}$ test. A p value of $\mathbf{0 . 0 5}$ or less was considered significant.

\section{Results}

CLINICAL FEATURES

A total of 170 adults with acute pneumonia were studied during March-December 1986. Of these, $15(8.8 \%)$ had $H$ influenzae isolated from cultures of blood or lung aspirate, or both. Eight were male $(53 \%)$ and seven female $(47 \%)$, with an age range of 
Table 1 Capsular serotypes of Haemophilus influenzae in adults with pneumonia

\begin{tabular}{lc}
\hline Type & No of cases \\
\hline Non-capsulate (non-typable) & 3 \\
Capsulate: & 4 \\
Type a & 7 \\
Type b & 1 \\
Type c & 15 \\
Total & \\
\hline
\end{tabular}

18-62 (mean 36.6) years, and all were Melanesian. In the total group of 170 patients there were $72 \%$ males and $28 \%$ females, with an age range of $12-70$ (mean 36) years. The difference in sex distribution between the patients with $H$ influenzae pneumonia and the total pneumonia group was not significant $(\mathrm{p}>0.2)$,

Chronic lung disease (chronic bronchitis or bronchiectasis, diagnosed on clinical and radiological criteria) was present in nine of the patients with $H$ influenzae pneumonia $(60 \%)$. In contrast, only $25 \%$ of the total group of patients with pneumonia had chronic lung disease $(p<0.02)$. A significant smoking history ( $>10$ cigarettes/day) was obtained in seven of the 15 patients $(47 \%)$ with $H$ influenzae pneumonia and in 107 of the $170(63 \%)$ in the total pneumonia group $(p>0.3)$.

\section{BACTERIOLOGY}

All 170 patients had blood cultures performed, and 144 underwent lung aspiration (the remaining 26 patients did not have lung aspiration because DJB was not available at the time when they presented).

Three of the 15 patients with $H$ influenzae pneumonia had positive blood and lung aspirate cultures, 11 had positive lung aspirate cultures only, and one had positive blood cultures only. In all 15 cases $H$ influenzae was the sole organism isolated. Type b was the commonest serotype isolated, accounting for seven of the 15 cases $(47 \%)$ (table 1$)$. Three patients had pneumonia due to non-typable $H$ influenzae, one of whom had positive blood cultures. The three other cases of bacteraemic $\boldsymbol{H}$ influenzae pneumonia were due to type a (two cases) and type b (one case).

Sputum cultures were obtained in 140 of the 170 cases. $H$ influenzae was isolated in 49 of the 140 patients $(35 \%)$. These 49 patients were examined to determine the relationship between sputum cultures positive for $\boldsymbol{H}$ influenzae and the results of cultures of blood and lung aspirate. In $55 \%$ of cases where $H$ influenzae was isolated from a sputum culture, another organism was isolated from a culture of blood or lung aspirate, or both (table 2). In only nine of 49 cases $(18 \%)$ where $H$ influenzae was grown from sputum was it also isolated from cultures of blood or lung aspirate.
Table 2 Number of sputum cultures positive for Haemophilus influenzae compared with results of blood and lung aspirate cultures

\begin{tabular}{lc}
\hline & $N o(\%)$ \\
\hline Sputum culture: $H$ influenzae isolated & $49(100)$ \\
Blood and lung aspirate cultures: & \\
$H$ influenzae isolated & $9(18)$ \\
Different organism isolated & $27(55)$ \\
Negative culture & $13(27)$ \\
\hline
\end{tabular}

\section{RADIOLOGY}

Lobar consolidation was present radiologically in $10 \overrightarrow{-}$ $(67 \%)$ of the 15 patients (unilobar in seven and bilobar in three), while five $(33 \%)$ showed $a_{\circ}$ bronchopneumonic pattern affecting predominantly both lower lobes. Lobar consolidation was present in $\vec{Z}$ three of the four bacteraemic patients; the fourtho had patchy bronchopneumonic changes confined to $\frac{\Phi}{7}$ the left lower lobe.

\section{TREATMENT AND OUTCOME}

Eight of the 15 patients with $H$ influenzae pneumonia were treated with penicillin (dose $8 \times 10^{6}$ units/day). Six other patients received ampicillin, and one was given chloramphenicol. All 15 patients recovered; the

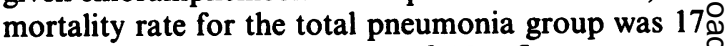
out of $170(10 \%)$. No cases of $H$ influenzae pneumonia were complicated by pleural effusion, lung $\stackrel{2}{\Rightarrow}$ abscess, or empyema.

\section{Discussion}

Haemophilus influenzae is an uncommon cause of pneumonia in adults. A 10 year study of adult pneumonia in three hospitals ${ }^{6}$ described 41 cases. The diagnosis was based on transtracheal aspirate alone in 3 16 of the cases. This technique of bacteriological diag-i nosis, while superior to sputum culture, may also be unreliable because of the tendency of $\boldsymbol{H}$ influenzae to colonise the lower respiratory tract in patients with chronic lung disease. The unreliability of sputum cul $\frac{D}{0}$ ture as a means of bacteriological diagnosis in patients with acute pneumonia has been shown in several studies. ${ }^{671213}$ This was confirmed in ourv study, in which $55 \%$ of cases where $H$ influenzae was isolated from sputum were found (from blood and ${ }^{\omega}$ lung aspirate cultures) to be due to another organism? Our 15 cases, collected during 10 months, were ale diagnosed from blood or percutaneous lung aspirate cultures, or both, which avoids the problem of colonisation.

Most studies of $\boldsymbol{H}$ influenzae in adults indicate

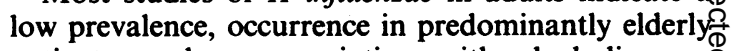
patients, and an association with alcoholism or chronic lung disease. ${ }^{3-1114-16}$ Our study confirmed the association with chronic lung disease; none of our 
patients, however, was alcoholic, and the age distribution and mean age were very similar to those for the total group of patients with pneumonia. Our patients were generally much younger than those with haemophilus pneumonia reported from developed countries but clearly there is a considerable difference in life expectancy between the different populations, with an average life expectancy in Papua New Guinea of $45-50$ years. While most cases $(12 / 15)$ were due to capsulate serotypes three were due to non-capsulate strains, showing that these are capable of producing invasive disease, including bacteraemia. Type $b$ was predominant in our series, as in other studies. $^{6-111415}$

Multilobar infiltrates, often involving the lower lobes of both lungs, are the predominant radiological pattern reported in $H$ influenzae pneumonia ${ }^{6} 16$ and were confirmed in this study, where lobar consolidation was present in $67 \%$ of cases. None of our patients had evidence of pleural effusion, in contrast to the relatively high incidence of this in other studies. $^{6716}$

There were no deaths due to $H$ influenzae pneumonia, though four patients were bacteraemic. The overall mortality in reported cases of bacteraemic $H$ influenzae pneumonia in adults is $34 \%{ }^{6}$ The successful treatment of $H$ influenzae pneumonia with penicillin, if given parenterally and in high doses, has been noted previously. ${ }^{6}$ Eight patients in our study were treated successfully with penicillin alone, despite the demonstration of in vitro resistance.

$H$ influenzae has become increasingly recognised as a cause of pneumonia in adults. The reasons for this are unclear, but may be related to declining immunity in adults to $H$ influenzae. It has been shown that up to $25 \%$ of healthy adults lack appreciable serum bactericidal activity against $H$ influenzae. ${ }^{17-20}$ Early and intensive treatment of $H$ influenzae infections with antibiotics may suppress or abolish the development of protective antibodies. If this proves to be correct, it would seem a valid explanation for the relatively high prevalence of $H$ influenzae pneumonia in Papua New Guinea, where the standard treatment for any febrile illness invariably includes the administration of parenteral penicillin.

In summary, in the course of 10 months 15 adults presented with $H$ influenzae pneumonia. Features of this group of patients included chronic lung disease, type b serotype infection, lobar consolidation, and a good response to antibiotic treatment, with no deaths.

We thank Denise Neagle and Ms Raka Ova for secretarial assistance in the preparation of the manuscript.

\section{References}

1 Smith DW. Hemophilus infections (Chapt.125).
In: Isselbacher $\mathrm{KJ}$, Adams RD, Braunwald $\mathrm{E}$, Petersdorf RG, Wilson JD, eds. Harrison's principles of internal medicine. New York: McGraw-Hill, 1980:650-1.

2 Hirschmann JV, Everett ED. Hemophilus influenzae infections in adults: report of nine cases and a review of the literature. Medicine (Baltimore) 1979;58:80-90.

3 Goldstein E, Daly AK, Seamans C. Haemophilus influenzae as a cause of adult pneumonia. Ann Intern Med 1967;66:35-40.

4 Berk SL, Holtsclaw SA, Wiener SL, Smith JK. Nontypable Hemophilus influenzae in the elderly. Arch Intern Med 1982;142:537-9.

5 Dworzack DL, Blessing LD, Hodges GR, Barnes WG. Hemophilus influenzae type $F$ pneumonia in adults. Am J Med Sci 1978;275:87-91.

6 Wallace RJ, Musher DM, Martin RR. Hemophilus influenzae pneumonia in adults. $A m \vec{M}$ Med 1978;64:87-93.

7 Levin DC, Schwarz MI, Matthay RA, LaForce FM. Bacteremic Hemophilus influenzae pneumonia in adults. A report of 24 cases and a review of the literature. Am J Med 1977;62:219-24.

8 Crowell $\mathrm{J}$, Loche SD. Primary Hemophilus influenzae pneumonia: report of four cases. Arch Intern Med 1954;93:921-4.

9 Kaplan NM, Braude AI. Hemophilus influenzae infection in adults. Arch Intern Med 1958;1010:515-8.

10 Johnson WD, Kaye D, Hook EW. Hemophilus influenzae pneumonia in adults. Report of five cases and review of the literature. Am Rev Respir Dis 1968;97:1112.

11 Everett ED, Rahm AE, Adaniga R, Stevens DL, McNitt TR. Haemophilus influenzae pneumonia in adults. JAMA 1977;238:319-21.

12 Barrett-Connor $\mathrm{E}$. The non value of sputum culture in the diagnosis of pneumococcal pneumonia. Am Rev Respir Dis 1971;103:845-8.

13 Fiala M. A study of the combined role of viruses, mycoplasmas and bacteria in adult pneumonia. $\mathrm{Am} \mathrm{J}$ Med Sci 1969;257:44-8.

14 Quintiliani R, Hymans PJ. The association of bacteremic Hemophilus influenzae pneumonia in adults with typeable stains. Am J Med 1971;50:781.

15 Tillotson JR, Lerner AM. Hemophilus influenzae bronchopneumonia in adults. Arch Intern Med 1968;121:428-30.

16 Pearlsberg J, Haggar AM, Saravolatz L, Becte GH, Popovich J. Hemophilus influenzae pneumonia in the adult. Radiographic appearance with clinical correlation. Radiology 1984;151:23-6.

17 Norden CW, Callerame ML, Baum J. Hemophilus influenzae meningitis in an adult. A study of bactericidal antibodies and immunoglobulins. $N$ Engl $J$ Med 1970;282:190-4.

18 Norden CW. Prevalence of bactericidal antibodies to Hemophilus influenzae, type b. J Infect Dis 1974;130:489-91.

19 Feigin RO, Richmond D, Howler MW, et al. Reassessment of the role of bactericidal antibody in Hemophilus influenzae infection. Am J Med Sci 1971;262:338-41.

20 Anderson P, Johnston RB, Smith DH. Human serum activities against Hemophilus influenzae type b. J Clin Invest 1972;51:31-4. 